\section{Histologist's original opinion compared with multidisciplinary team in determining diagnosis in interstitial lung disease}

\section{ABSTRACT}

Guidelines recommend that multidisciplinary interstitial lung disease meeting (ILD MDT) decisions become the gold standard for diagnosis, replacing the histologist from this position, and identify this as requiring supportive evidence. We have compared diagnoses from lung biopsy material made by expert histologists with the subsequent consensus opinion from a properly constituted ILD MDT in 71 consecutive patients referred to a regional thoracic unit. MDT changed the original histological diagnoses in 30\% (95\% CI $19.3 \%$ to $41.6 \%$ ) and strengthened the diagnoses from probable to confident in a further 17\% (95\% CI 9.1\% to $27.7 \%$ ). The assessment of hypersensitivity pneumonitis, non-necrotising granulomas and organising pneumonia accounted for the majority of the changes.

\section{INTRODUCTION}

The multidisciplinary team (MDT) consensus has replaced the histopathologist as the gold standard in the diagnosis of specific interstitial lung diseases (ILDs), ${ }^{1-3}$ an opinion endorsed by the National Institute for Health and Care Excellence (NICE) UK, which also defined the core members to include a specialist radiologist, histopathologist, clinician and clinical nurse specialist. ${ }^{4}$ There is, however, a lack of evidence related to this change in a wide range of ILDs seen in clinical practice. We have compared diagnoses from lung biopsy material made by expert histologists with the subsequent consensus opinion from a properly constituted ILD MDT in 71 consecutive patients referred to a regional thoracic unit, when the referring clinician was unable to make a specific diagnosis without this. The setting and methods are shown in the online supplement.

\section{RESULTS}

Seventy-one patients had qualifying videoassisted thoracoscopic surgery (VATS) biopsies. The results of the MDT diagnosis and the numbers supported by firm conclusions from the histology report are shown in table 1 . In 21 patients (30\%, $95 \%$ CI $19.3 \%$ to $41.6 \%$ ), the MDT diagnosis differed significantly from the histology report, and in a further 12
(17\%, 95\% CI $9.1 \%$ to $27.7 \%)$ MDT strengthened the diagnosis from probable to confident. Weighted Cohen's kappa statistic for agreement was insignificant; $\kappa=0.034(p=0.15 ; 95 \% \mathrm{CI}-0.3$ to 0.1$)$ for 71 histology/MDT pairs. There was good consistency for patients with a histological diagnosis of usual interstitial pneumonitis (UIP). The histology was non-specific in one patient with an MDT diagnosis of acute interstitial pneumonitis with a background of UIP; in a further four, the histological diagnosis included the possibility of UIP. MDT had sufficient evidence to further characterise UIP to idiopathic pulmonary fibrosis (IPF) (6), collagen vascular disease associated (1), drug induced (1) or due to hypersensitivity pneumonitis (HP) (1). MDT made a confident diagnosis of non-specific interstitial pneumonitis (NSIP) in eight cases and probable NSIP in one case. The histology identified non-specific fibrosis in three and organising pneumonia in a further two, who on MDT review had NSIP in addition to the organising pneumonia. MDT identified the cause as chronic HP and rheumatoid arthritis in one each. There were eight patients with an MDT diagnosis of HP. These posed a particular problem to the histologists whose initial diagnoses were non-specific in three and included HP in differential diagnoses in a further four. Four patients had necrotising granulomas, and MDTconfirmed diagnoses of rheumatoid lung (1), sarcoidosis (1) and no ILD (2). Organising pneumonia was the primary histological diagnosis in two and present

in a further two. MDT identified underlying interstitial disease in all of them, one with a confident diagnosis of NSIP, one with benign lymphoid hyperplasia and two with a differential diagnosis of $\mathrm{HP} /$ NSIP. The ILD MDT made a specific diagnosis in five patients whose biopsies were reported as non-specific fibrosis; NSIP in 2, UIP in 2, HP (1), and in 2 in whom the original report was resolving pneumonia (both HP). The ILD MDT thought that there was insufficient evidence to diagnose any ILD in eight patients. Four had nodules, diagnosed as rheumatoid nodules without ILD (2), apical cap (1) and necrotising granuloma unspecified (1). In the remaining four, there was evidence of fibrosis that was thought to be postinfectious.

There was a lack of specific probable diagnoses pre-VATS for many of the patients. Of the eight patients with an ILD MDT diagnosis of HP, four were thought to have unspecified ILD and one was thought to have sarcoidosis. HP was in the pre-VATS differential for only two patients, the ILD MDT-confirmed NSIP due to HP in one and IPF unrelated to hypersensitivity in the other. Sarcoidosis was the pre-VATS diagnosis in seven patients, confirmed in only two, thesaurosis in one, $\mathrm{HP}$ in one, langerhans cell granulomatosis in one and IPF in two.

There were two postoperative deaths, one from a postoperative myocardial infarction and one from an acute exacerbation of IPF. A third patient with pleuroparenchymal fibroelastosis had a postoperative bleeding requiring a thoracotomy for control.

Table 1 A comparison of the diagnosis made by ILD MDT with the diagnosis on the original histopathology report

\begin{tabular}{lll}
\hline Diagnosis & $\begin{array}{l}\text { MDT numbers } \\
\text { (probable) }\end{array}$ & $\begin{array}{l}\text { Histology* numbers } \\
\text { (probable) }\end{array}$ \\
\hline Usual interstitial pneumonitis & 21 & $16(4)$ \\
Non-specific interstitial pneumonitis & $8(1)$ & 4 \\
Desquamative interstitial pneumonitis/respiratory & 6 & 4 \\
bronchiolitis ILD & & 2 (4) \\
Hypersensitivity pneumonitis & 8 & 0 \\
Pleuroparenchymal fibroelastosis & 1 & 1 \\
Mixed dust fibrosis & 1 & $3(1)$ \\
Sarcoidosis & 5 & 0 \\
Thesaurosis & 1 & 2 \\
Langerhans cell granulomatosis & 2 & 0 \\
Inflammatory myoblastic tumour & 2 & 1 \\
Lymphoproliferative hyperplasia benign & 1 & 3 \\
TB & 4 & 2 \\
Organising pneumonia & $(2)$ & 1 \\
No ILD & 8 & \\
\hline *Includes probable and definite diagnoses only. Non-specific diagnoses are excluded from this column. \\
ILD, interstitial lung disease; MDT, multidisciplinary team.
\end{tabular}




\section{DISCUSSION}

An ILD MDT review by a specialist team of histopathologists, radiologists, clinicians and a clinical nurse specialist provided significant extra benefits in terms of a precise diagnosis in patients biopsied with ILD, in whom the referring physician thought that a diagnosis was not possible without a biopsy. MDT changed the diagnosis in 30\% and strengthened the diagnosis from probable to confident in a further $17 \%$. The interpretation of the histopathology changed as a result of team discussion and presentation of clinical and radiology data by experts in ILD at the meeting, rather than changing the histological features seen in the biopsies. The benefits of MDT's are not surprising as the histologist is limited by sampling and information on distribution, the radiologist by resolution and both by limited access to clinical history, rate of disease progression, relevant exposures and the presence or absence of collagen vascular disease. The MDT decision might also be incorrect. Walsh et $a l^{5}$ compared agreement between seven expert ILD MDTs and found good agreement for IPF and connective disease-associated ILD, with weighted kappas between 0.6 and 0.73 ; the kappas for NSIP and HP were much worse. In our study the diagnosis of HP was particularly helped by the multidisciplinary discussion, a group that other MDTs have found problematical. ${ }^{5} \quad 6$ Finding the causes for organising pneumonia and granuloma was also an important contribution. Previous studies have shown poor reproducibility of a diagnosis made on radiology or histology alone. 78

Whole group discussions have improved consensus opinions after independent diagnoses of academic and community clinicians, radiologists and histopathologists.
Lung biopsy is not without serious risks and morbidity. The value of surgical lung biopsies for those with less classical CTs is questioned when the likely diagnosis is UIP. $^{10}$

\section{PS Burge, J Reynolds, S Trotter, GA Burge, G Walters}

Interstitial Lung Disease Service, Birmingham Heartlands Hospital, Birmingham, UK

Correspondence to Professor PS Burge, Interstitia Lung Disease Service, Birmingham Heartlands Hospital, Birmingham B9 5SS, UK; sherwood.burge@doctors.org. uk

Collaborators Specialist Radiology team for interstitia lung disease (ILD): JR, Dr Madava Djearaman, Dr Shahid Hussain and Dr Ed Hoey. Specialist histology team for ILD: Dr ST, Dr Gerald Langman, Sherin Payyappilly and Praba Naidoo. Thoracic surgeons supporting lung biopsy service: Mr Maninder Kalkat, Mr Richard Steyn, Mr Pala Rajesh, Mr Ehab Bishay and Professor Babu Naidu. Clinical team: PSB, GW, Dr Salman Ghani, Dr Dimitrina Petkova, Dr Alastair Robertson, Dr J Jayakumar and GAB.

Contributors All authors are core members of the ILD multidisciplinary team and have contributed to the paper. Others with lesser contributions are acknowledged.

Competing interests None.

Provenance and peer review Not commissioned; externally peer reviewed.

- Additional material is published online only. To view please visit the journal online (http://dx.doi.org/10. 1136/thoraxjnl-2016-208776)

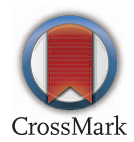

To cite Burge PS, Reynolds J, Trotter S, et al. Thorax 2017:72:280-281.

Received 14 April 2016

Revised 6 October 2016

Accepted 13 October 2016

Published Online First 4 November 2016

Thorax 2017;72:280-281.

doi:10.1136/thoraxjnl-2016-208776

\section{REFERENCES}

1 Wells AU. Histopathologic diagnosis in diffuse lung disease: an ailing gold standard. Am Resp Crit Care Med 2004;170:828-9.

2 Wells $\mathrm{AU}$, Hirani $\mathrm{N}$, on behalf of the British Thoracic Society. Interstitial Lung Disease Guideline Group, a subgroup of the British Thoracic Society Standards of Care Committee, in collaboration with the Thoracic Society of Australia and New Zealand and the Irish Thoracic Society. Thorax 2008:63:v1-58.

3 Raghu G, Collard HR, Egan JJ, et al. An official ATS/ERS/JRS/ALAT statement: idiopathic pulmonary fibrosis: evidence-based guidelines for diagnosis and management. Am J Respir Crit Care Med 2011;183:788-824.

4 National Institute for Health and Care Excellence. Idiopathic pulmonary fibrosis in adults: diagnosis and management [CG163]. London: NICE, 2013.

5 Walsh SL, Wells AU, Desai SR, et al. Multicentre evaluation of multidisciplinary team meeting agreement on diagnosis in diffuse parenchymal lung disease: a case-cohort study. Lancet Respir Med 2016:4:557-65.

6 Jo HE, Corte TJ, Moodley Y, et al. Evaluating the interstitial lung disease multidisciplinary meeting: a survey of expert centres. BMC Pulm Med 2016:16:22.

7 Walsh SLF, Calandriello L, Sverzellati N, et al. on behalf of The UIP Observer Consort. Interobserver agreement for the ATS/ERS/JRS/ALAT criteria for a UIP pattern on CT. Thorax 2016:71:45-51.

8 Thomeer M, Demedts M, Behr J, et al. Idiopathic Pulmonary Fibrosis International Group Exploring $\mathrm{N}$-Acetylyysteine I Annual (IFIGENIA) study group. Eur Respir J. 2008;31:585-91.

9 Flaherty KR, Andrei AC, King TE Jr, et al. Idiopathic interstitial pneumonia: do community and academic physicians agree on diagnosis? Am I Respir Crit Care Med 2007;175:1054-60.

10 Quadrelli S, Molinari L, Ciallella L, et al. Radiological versus histopathological diagnosis of usual interstitial pneumonia in the clinical practice: does it have any survival difference? Respiration 2010;79:32-7. 